\title{
Heart pacemaker wear life model based on frequent properties and life distribution*
}

\author{
Qiao-Ling Tong ${ }^{1}$, Xue-Cheng Zou ${ }^{1}$, Jin Tang ${ }^{2}$, Heng-Qing Tong ${ }^{2}$ \\ ${ }^{1}$ Department of Electronic Science \& Technology, Huazhong University of Science \& Technology, Wuhan, China; \\ ${ }^{2}$ Department of Mathematics, Wuhan University of Technology, Wuhan, China. \\ Email: qltong@gmail.com
}

Received 4 January 2010; revised 20 January 2010; accepted 25 January 2010.

\begin{abstract}
The lifetime of heart pacemaker is important for patient and researcher. To forecast the lifetime of heart pacemakers we must determine its distribution regularity. In this paper, a heart pacemaker wear life model is introduced by using frequency property, and a life distribution is presented. The parametric properties of the density are studied. The moment estimator with negative order is used, which is just the maximum likelihood estimator. A new method of parameter estimate, $F$ estimate of parameter, is proposed. This method is suitable to both truncated samples and complete samples, whether or not the distribution can be transformed into a standard distribution without any parameters.
\end{abstract}

Keywords: Heart Pacemaker Life Distribution; Frequency; Negative Order Moment Estimator; Maximum Likelihood Estimate; $F$ Estimate

\section{INTRODUCTION}

The heart pacemakers (HPM) have more and more extensive clinical applications. Almost all users of HPM worry the lifetime of HPM. If some time his HPM stops work out of the blue, that will be serious trouble. Postponing or advancing to replace or to repair HPM is not suitable. So forecasting the lifetime of heart pacemaker is important for patient and researcher.

Extending HPM lifetime is the significant aim to reduce the cost of cardiac pacing [1]. The factors which impact HPM Life content: "innate" factors and "acquired" factors (such as electromagnetic fields, drugs, etc.) [2], but the former is more important. The innate factors include many aspects which will be introduced as follows. The battery capacity is decided by battery energy and battery cubage. For the same functional circuit, increasing battery capacity will enhance its capacity,

*The research was supported by the National Natural Science Foundation of China (30570611, 60773210). decrease pacing power and prolong the HPM life. Internal faradic current is a significant, independent factors related to battery life. Low internal faradic current is an important role to save battery life [2,3]. Although there is a little literature to introduce the relativity between internal faradic current and battery life, some scholars for this study think that internal faradic current has a major impact on HPM life and maybe is greater than the battery capacity and output energy. Markewitz, etc. [3] found that decreasing the internal faradic current consumption enables to reduce overall current consumption of HPM and gain a longer life, which approves that the internal faradic current is a foremost factor of influencing HPM life. Pacing frequency also has a marked influence on HPM life, and its level of energy consumption is proportional to the HPM. HPM electrode function is the weakest link in the system, which is also the most important factors to determine the security and life of HPM system [4]. After the HPM pulse generator (Pulse generator, PG) was designed, the battery capacity and current consumption has been identified and remain constant. Improving the wire electrode function is the only way to prolong HPM lifetime. Electrodes have many factors which will affect the HPM life. According to Ohm's law, the high impedance (> $1000 \mathrm{ohm}$ ) can reduce the loss current, increasing the HPM life.

Lead is also necessary. The main function of electrode lead is transmitting electricity, but its mechanism capability is important for assuring the life of pacemaker. The capability mostly lies on the material of insulating surface. Silica gel may be the best choice, which has been used over past 30 years. The resistance of lead rest with its length. The length of electrode with double pole is two times compared with unipolar one. In the theory, electrode with double pole uses more energy. Inductive circuit and pacing with multi-cavity or double cavity wastes more energy than single cavity and inductive circuit.

In this paper, a new heart pacemaker life distribution is presented according to relative influence factors. The 
heart pacemaker may be worn or damaged after working for a long time, therefore, its vibration and frequency may be changed. The frequency of a heart pacemaker can be recorded by a frequency record meter. The heart pacemaker life and its reliability can be investigated by the analysis of the heart pacemaker frequency.

Let $A_{i}^{+} i=1, \cdots, n$ be the amplitude values of the frequency of a normal heart pacemaker, $A_{i} i=1, \cdots, n$ be that of the damaged heart pacemaker. We define the frequency parameter $k$ :

$$
k=\frac{1}{n-1} \sum_{i=1}^{n} \frac{A_{i}}{A_{i}^{+}}
$$

Experiments have shown that we can deduce the level of the wear or the damage of a heart pacemaker according to the analysis of the frequency parameter $k$. When $k>k_{\max }$, the heart pacemaker can not work normally. The value of the frequency parameter increases as the work time of a heart pacemaker increases. In general, we suppose $k=r t$, then, we will get $t=\frac{k}{r}$, where $r$ denotes the change rate of $k$. It may be affected by many independent little factors such as capability of battery, the function of electrode, lead, material and so on. We suppose that $r$ is a random variable with normal distribution with definition $r>0$ :

$$
f_{r}(r)=\frac{C}{\sqrt{2 \pi} \sigma} \exp \left(-\frac{(r-\bar{r})^{2}}{2 \sigma^{2}}\right), \quad r>0
$$

From $\int f_{r}(r) d r=1$, we know $C=\frac{1}{\phi(\bar{r} / \sigma)}$, where $\phi$ is the distribution function of standard normal.

Let $t$ be the heart pacemaker wear life, $T=k_{\max } / r$, then $t$ is also a random variable. The density function of heart pacemaker wear life distribution is

$$
f(r)=\frac{C}{\phi(\bar{r} / \sigma) \sqrt{2 \pi} \sigma t^{2}} \exp \left(-\frac{(k-\bar{r} t)^{2}}{2 \sigma^{2} t^{2}}\right), \quad r>0
$$

When $k=k_{\max }$, its distribution function is

$$
F(t)=\frac{1-\Phi(k /(\sigma t)-\bar{r} / \sigma)}{\Phi(\bar{r} / \sigma)}
$$

Now, we particularly investigate statistical property of heart pacemaker wear life distribution. First, we see the graph of $f(t)$. Put $t \rightarrow 0^{+}$, then $f(t) \rightarrow 0^{+}$, because $t^{2} \exp \left(-\frac{(k /(\sigma t)-\mu / \sigma)^{2}}{2}\right) \rightarrow+\infty$, put $t \rightarrow+\infty$, $f(t) \rightarrow 0^{+}$. Differentiate $f(t)$, then

$$
f^{\prime}(t)=f(t)\left[-\frac{2}{t}+\frac{k(k / t-\bar{r})}{t^{2} \sigma^{2}}\right]
$$

The solution of equation $f^{\prime}(t)=0$ is

$$
t=\frac{k}{4 \sigma^{2}}\left(-\bar{r} \pm \sqrt{\bar{r}^{2}+8 \sigma^{2}}\right)
$$

Second, we see the change of failure rate $\lambda$.

$$
\begin{aligned}
\lambda(t) & =\frac{f(t)}{1-F(t)} \\
& =\frac{k \phi(\bar{r} / \sigma)}{\phi(\bar{r} / \sigma) \sqrt{2 \pi} \sigma t^{2}} \times \frac{\exp \left(-(k / t-\bar{r})^{2} /\left(2 \sigma^{2}\right)\right)}{[\phi(\bar{r} / \sigma)-1+\phi(k / t \sigma)-\bar{r} / \sigma]}
\end{aligned}
$$

Let

$$
g(t)=t^{2} \exp \left(\frac{1}{2 \sigma^{2}}\left(\frac{k}{t}-\bar{r}\right)^{2}\right)\left[\phi\left(\frac{\bar{r}}{\sigma}\right)-1+\phi\left(\frac{k}{t \sigma}-\frac{\bar{r}}{\sigma}\right)\right]
$$

then $\lambda(t)=c / g(t)$, where $C$ is a constant. Because

$$
\lim _{t \rightarrow 0^{+}} g(t)=+\infty
$$

and

$$
\begin{aligned}
\lim _{t \rightarrow+\infty} g(t)= & \exp \left(\frac{\bar{r}^{2}}{2 \sigma^{2}}\right) \times \lim _{t \rightarrow+\infty} \frac{\phi(\bar{r} / \sigma)-1+\phi(k / t \sigma-\bar{r} / \sigma)}{t^{-2}} \\
& =\exp \left(\frac{\bar{r}^{2}}{2 \sigma^{2}}\right) \times \lim _{t \rightarrow+\infty}\left[\frac{k t}{2 \sqrt{2 \pi} \sigma} \exp \left(-\frac{1}{2}\left(\frac{k}{t \sigma}-\frac{\bar{r}}{\sigma}\right)^{2}\right)\right] \\
& =+\infty
\end{aligned}
$$

We have $\lim _{t \rightarrow 0^{+}} \lambda(t)=\lim _{t \rightarrow \infty} \lambda(t)=0$. The graph of $\lambda(t)$ is also located on the first quadrate. Its left limit is origin and its right asymptotic line is $t$ axis.

\section{MOMENT ESTIMATE WITH NEGATIVE ORDER FOR COMPLETE SAMPLE}

When we investigate the parameter estimate of the density function $f(t)$ of heart pacemaker wear life distribution, we notice that it has two independent parameters only. Let $a=k / \sigma, b=\bar{r} / \sigma$, then the density function of heart pacemaker wear life distribution is

$$
f(t)=\frac{a}{\sqrt{2 \pi} \Phi(b) t^{2}} \exp \left(-\frac{1}{2}\left(\frac{a}{t}-b\right)^{2}\right), \quad t>0
$$

In Eq.3, $f(t)$ has three parameters $k, \sigma, \bar{r}$. They are independent in the physical process, but they are dependent in the density function. We can estimate $a, b$ 
from Eq.10. We need to estimate $k$ in addition, then we can estimate $\sigma, \bar{r}$.

First, we consider moment estimator. For common moment estimator $E\left(t^{j}\right), j=1,2, \cdots$, we can not calculate its integration. If we make use of moment estimator with negative order $E\left(t^{j}\right), j=-1,-2, \cdots$, the integration can be calculated easily.

$$
\begin{aligned}
E T^{-1} & =\int_{0}^{+\infty}\left[\frac{a}{\sqrt{2 \pi} \Phi(b) t^{3}} \exp \left(-\frac{1}{2}\left(\frac{a}{t}-b\right)^{2}\right)\right] d t \\
& =\frac{1}{a \Phi(b)} \int_{0}^{+\infty}\left[(y+b) \frac{1}{\sqrt{2 \pi}} \exp \left(-\frac{1}{2} y^{2}\right)\right] d y \\
& =\frac{b}{a}+\frac{1}{\sqrt{2 \pi} a \Phi(b)} \exp \left(-\frac{1}{2} b^{2}\right) \\
(y & \left.=\frac{a}{t}-b\right) \\
E T^{-2}= & \int_{0}^{+\infty}\left[\frac{a}{\sqrt{2 \pi} \Phi(b) t^{4}} \exp \left(-\frac{1}{2}\left(\frac{a}{t}-b\right)^{2}\right)\right] d t \\
= & \frac{1}{a^{3} \Phi(b)} \int_{-b}^{+\infty}\left[(y+b)^{2} \frac{1}{\sqrt{2 \pi}} \exp \left(-\frac{1}{2} y^{2}\right)\right] d y \\
= & \frac{b^{2}+1}{a}+\frac{b}{\sqrt{2 \pi} a^{2} \Phi(b)} \exp \left(-\frac{1}{2} b^{2}\right)
\end{aligned}
$$

Let $t_{1}, \cdots, t_{n}$ be i.i.d. sample data of the heart pacemaker wear life. We make use of the sample moment to estimate the population moment. The difference is the sign of the order of moment. From the system of equations

$$
\begin{aligned}
& s_{1}=\frac{1}{n} \sum_{i=1}^{n} \frac{1}{t_{i}}=\frac{b}{a}+\frac{1}{\sqrt{2 \pi} a \Phi(b)} \exp \left(-\frac{1}{2} b^{2}\right) \\
& s_{1}=\frac{1}{n} \sum_{i=1}^{n} \frac{1}{t_{i}^{2}}=\frac{b^{2}+1}{a}+\frac{1}{\sqrt{2 \pi} a^{2} \Phi(b)} \exp \left(-\frac{1}{2} b^{2}\right)
\end{aligned}
$$

we have

$$
\left\{\begin{array}{l}
b=\frac{1}{s_{1}}\left(a s_{2}-\frac{1}{a}\right) \\
a=\frac{1}{s_{1}}\left[b+\frac{1}{\sqrt{2 \pi} a \Phi(b)} \exp \left(-\frac{1}{2} b^{2}\right)\right]
\end{array}\right.
$$

Moreover we can obtain the solution $\widehat{a}$ and $\hat{b}$ from Eq.14. They are the moment estimate of $a$ and $b$.

Second, we consider the maximum likelihood estimator of parameters. The likelihood function is

$$
L(a, b)=\left(\frac{a}{\sqrt{2 \pi} \Phi(b)}\right)^{n} \frac{1}{\prod_{i=1}^{n} t_{i}^{2}} \exp \left(-\frac{1}{2}\left(\frac{a}{t_{i}}-b\right)^{2}\right)
$$

Taking derivation of $L(a, b)$, we have

$$
\begin{aligned}
\frac{\partial L}{\partial a} & =n\left(\frac{a}{\sqrt{2 \pi} \Phi(b)}\right)^{n-1} \frac{1}{\sqrt{2 \pi} \Phi(b)} \frac{1}{\prod_{i=1}^{n} t_{i}^{2}} \exp \left(-\frac{1}{2} \sum_{i=1}^{n}\left(\frac{a}{t_{i}}-b\right)^{2}\right) \\
& +\left(\frac{a}{\sqrt{2 \pi} \Phi(b)}\right)^{n} \frac{1}{\prod_{i=1}^{n} t_{i}^{2}} \exp \left(-\frac{1}{2} \sum_{i=1}^{n}\left(\frac{a}{t_{i}}-b\right)^{2}\right) \times\left[-\sum_{i=1}^{n}\left(\frac{a}{t_{i}}-b\right)^{2} \frac{1}{t_{i}}\right] \\
& =0
\end{aligned}
$$

and

$$
\begin{aligned}
\frac{\partial L}{\partial b} & =n\left(\frac{a}{\sqrt{2 \pi} \Phi(b)}\right)^{n-1} \frac{a \Phi^{\prime}(b)}{\sqrt{2 \pi} \Phi(b)} \frac{1}{\prod_{i=1}^{n} t_{i}^{2}} \exp \left(-\frac{1}{2} \sum_{i=1}^{n}\left(\frac{a}{t_{i}}-b\right)^{2}\right) \\
& +\left(\frac{a}{\sqrt{2 \pi} \Phi(b)}\right)^{n} \frac{1}{\prod_{i=1}^{n} t_{i}^{2}} \exp \left(-\frac{1}{2} \sum_{i=1}^{n}\left(\frac{a}{t_{i}}-b\right)^{2}\right) \times\left[-\sum_{i=1}^{n}\left(\frac{a}{t_{i}}-b\right)^{2}\right] \\
& =0
\end{aligned}
$$

Simplifying Eq.16 and Eq.17, we have

$$
\left\{\begin{array}{l}
a^{2} s_{2}-a b s_{1}=1 \quad \Leftrightarrow \quad b=\frac{1}{s_{1}}\left(a s_{2}-\frac{1}{a}\right) \\
s_{1} a=b+\frac{\Phi^{\prime}(b)}{\Phi(b)} \Leftrightarrow \quad a=\frac{1}{s_{1}}\left[\quad b+\frac{e^{\left(-\frac{1}{2} b^{2}\right)}}{\sqrt{2 \pi} a \Phi(b)}\right]
\end{array}\right.
$$

Noting that Eq.18 is just Eq.19, we know that the moment estimator with negative order of heart pacemaker wear life distribution is just its maximum likelihood estimator. It is more reasonable to take the moment estimator with negative order for heart pacemaker wear life distribution.

\section{PARAMETER ESTIMATE FOR TRUNCATED TESTING}

Life testing is often truncated by the given size of samples, because the time and the cost of testing are limited. Suppose $n$ products are taken as life testing. We have observed $m$ products which have been failures. $t_{1} \leq t_{2} \leq \cdots \leq t_{m}$ are $m$ preceding order statistics of products life. For exponential distribution, Weibull distribution, normal and lognormal distribution, we can give parameter estimator in truncated testing. These distributions can be transformed into standard distributions without any parameter. According to the order statistic of the standard distribution, we can compute its expectation, variance and covariance. Then, we can obtain the Best Linear Unbiased Estimation by the least square method. But we can not do it in this way for heart pacemaker wear life distribution. The reason is it can not be transformed into any standard distribution without any parameter. The maximum likelihood esti- 
mate of heart pacemaker wear life distribution from the order statistics of the truncated samples is not certain to converge, because its density function does not satisfy some of the convergency conditions.

In this paper, a new statistical method, $F$ estimate of parameter, is proposed. It is suitable to any distribution whether or not it can be transformed into a standard distribution. What is more, it has no definition of sample size. We can consider the problem in this way. For the lives of $n$ products in life testing, we have observed $m$ preceding lives of products. We have not observed $n-m$ late lives of products but they are existent. For the empirical distribution function of life $t$, we have observed $F_{n}\left(t_{1}\right)=1 / n, \cdots, F_{n}\left(t_{m}\right)=m / n$. We have not observed the late values, but they also exist. From Glivenko-Cantelli Theorem, we know that empirical distribution function of random variable with probability one converges to its distribution function uniformly. That is,

$$
P\left\{\lim _{n \rightarrow \infty}\left(\sup _{-\infty<t<+\infty}\left|F_{n}(t)-F(t)\right|\right)=0\right\}=1
$$

Therefore we can obtain $m$ approximate equations $F_{n}\left(t_{i}\right)=F\left(t_{i}\right), i=1, \cdots, m$, i.e.,

$$
\left\{\begin{array}{l}
F\left(t_{i}\right)=\frac{1}{n} \\
F\left(t_{2}\right)=\frac{2}{n} \\
\cdots \cdots \\
F\left(t_{m}\right)=\frac{m}{n}
\end{array}\right.
$$

The parameters to be estimated are contained in the system of Eq.20. The problem is changed into solving a nonlinear regression model. Solving the model by the least square method, we can obtain the parameters to be estimated. We call it $F$ estimate of parameter. Obviously, this method is reliable in theory, simple in computation and extensive in application.

From [5], we know that heart pacemaker wear life distribution is

$$
F(t)=\frac{1-\Phi\left(\frac{a}{t}-b\right)}{\Phi(b)}
$$

The regression equations are

$$
\frac{1-\Phi\left(\frac{a}{t_{i}}-b\right)}{\Phi(b)}=\frac{i}{n}, \quad i=1, \cdots, m
$$

Making use of the least square method, we need to seek the minimum by changing parameters $a, b$ :

$$
Q(a, b)=\sum_{i=1}^{m}\left(\frac{1-\Phi\left(\frac{a}{t_{i}}-b\right)}{\Phi(b)}-\frac{i}{n}\right)^{2} \rightarrow \min
$$

Or we solve the following system of equations:

$$
\begin{aligned}
& \sum_{i=1}^{m}\left\{\left[\frac{1-\Phi\left(\frac{a}{t_{i}}-b\right)}{\Phi(b)}-\frac{i}{n}\right] \frac{1}{t_{i}} \exp \left(-\frac{1}{2}\left(\frac{a}{t_{i}}-b\right)^{2}\right)\right\}=0 \\
& \sum_{i=1}^{m}\left\{\left[\frac{1-\Phi\left(\frac{a}{t_{i}}-b\right)}{\Phi(b)}-\frac{i}{n}\right]\right. \\
& =0
\end{aligned}
$$

Of course, this method is also suitable to complete samples, that is $m=n$.

\section{COMPUTATION EXAMPLE AND COMPARISON OF PRECISION}

In order to investigate the properties of heart pacemaker wear life distribution carefully, and test the new statistical method, $F$ estimate of parameter, we compute examples on a computer. The parameters $a, b$ are given and random numbers are generated and are sequenced in terms of Eq.10. Using these data, first we compute the moment estimators with negative order (and also the maximum likelihood estimators) $\hat{a}_{m}, \hat{b}_{m}$ based on Eq.14 or Eq.18. Second, we compute the $F$ estimators of parameters $\hat{a}_{F}, \hat{b}_{F}$ using Eq.23. Third, we take $m$ preceding data and compute the $F$ estimators of parameters $\hat{a}_{F 1}, \hat{b}_{F 1}$ according to Eq.23 also. Compared them repeatedly by changing sample size $m$, parameters $a, b$ and truncated number $m$.

From the results of comparison, we know that it is robust to take absolute value in Eq.23.

$$
Q(a, b)=\sum_{i=1}^{m}\left|\frac{1-\Phi\left(\frac{a}{t_{i}}-b\right)}{\Phi(b)}-\frac{i}{n}\right| \rightarrow \min
$$

We take the Powell algorithm improved by Sargent to 
compute Eq.24 and the bisection method to compute Eq.14.

When data are small samples, we take sample size $n=20$, truncated number $m=10$, initial parameters $a=0.9, b=3.0$. The computed results are in Table 1 . The random data are generated 5 times and computations are repeated $k=5$ times. When the random data are generated, their seeds are different. First of all, these seeds are generated as random data independently. If it is necessary to calculate a great number of random data, we can change the modules of pseudorandom data yet. Using this method, the repeated number $k$ can be very large. We can calculate the empirical distribution function of the statistics $a_{m}, b_{m}, a_{F}$ and $b_{F}$. Therefore, we can draw up the distribution function tables of the statistics for given precision according to GlivenkoCantelli Theorem. In any case, we are satisfied with the computed results in Table 1 , because the sample size is so small.

When data are common samples, we take sample size $n=100$, truncated number $m=50$, initial parameters also use $a=9.0, b=3.0$. Computed results are in Table 2 . Of course, they are more accurate and also make us satisfied.

\section{CONCLUSIONS}

More and more functions are gathered in heart pacemaker. It is used more frequently along with the development ofbiomedical engineering. Extending and forecasting the lifetime of heart pacemaker is very important to user. In this paper, we introduced a heart pacemaker wear life model by making use of the frequency property, and deduced its life distribution. The parametric properties of the distribution density are interesting because its moment

Table 1. Computed results with $\mathrm{n}=20, \mathrm{~m}=10, \mathrm{a}=0.9, \mathrm{~b}=3.0$.

\begin{tabular}{ccccccc}
\hline$k$ & $a_{m}$ & $b_{m}$ & $a_{F}$ & $b_{F}$ & $a_{F 1}$ & $b_{F 1}$ \\
\hline 1 & 9.746 & 3.403 & 8.789 & 3.144 & 6.541 & 2.223 \\
2 & 8.295 & 2.711 & 9.853 & 3.228 & 6.067 & 1.804 \\
3 & 8.298 & 2.523 & 7.662 & 2.394 & 7.740 & 2.331 \\
4 & 10.675 & 3.712 & 13.032 & 4.629 & 6.980 & 2.223 \\
5 & 9.362 & 3.489 & 9.930 & 3.836 & 6.112 & 2.223 \\
\hline
\end{tabular}

Table 2. Computed results with $n=100, m=50, a=9.0, b=3.0$.

\begin{tabular}{ccccccc}
\hline$k$ & $a_{m}$ & $b_{m}$ & $a_{F}$ & $b_{F}$ & $a_{F 1}$ & $b_{F 1}$ \\
\hline 1 & 10.491 & 3.544 & 10.504 & 3.568 & 11.210 & 3.823 \\
2 & 9.787 & 3.281 & 9.628 & 3.223 & 9.547 & 3.187 \\
3 & 9.337 & 3.086 & 8.809 & 2.890 & 8.127 & 2.641 \\
4 & 9.750 & 3.340 & 9.502 & 3.261 & 7.710 & 2.485 \\
5 & 9.417 & 3.318 & 8.957 & 3.152 & 9.046 & 3.178 \\
\hline
\end{tabular}

estimator with negative order is just its maximum likelihood estimator. We also propose an $F$ estimate of parameter that is suitable to both truncated samples and complete samples. Through computation example and comparison of precision, we can get satisfying results. That means whether or not the distribution can be transformed into a standard distribution without any parameters, we can estimate the parameters in truncated data sample.

\section{ACKNOWLEDGEMENTS}

We would like to thank the experts who take their time to check, approve this paper and give us valuable suggestions.

\section{REFERENCES}

[1] Oham, O.J. and Danilovic, D. (1997) Improvements in pacemaker energy consumption and functional capability: Four decades of progress. Pacing and Clinical Electrophysiology, 20, 2-9.

[2] Kindermann, M., Schwaab, B., Berg, M. and Fröhlig, G. (2001) Longevity of dual chamber pacemaker: Device and patient related determiants. Pacing and Clinical Electrophysiology, 24(5), 810-815.

[3] Markewitz, A., Kronski, D., Kammeyer, A., Kaulbach, H., Weinhold, C., Doering, W. and Reichart, B. (1995) Determinants of dual chamber pulse generators longevity. Pacing and Clinical Electrophysiology, 18(12), 21162120.

[4] Crossley, G.H., Jeffrey, A.B., Reynolds, D., William, S., Johnson, W.B., Howard, H. and Lisa, T. (1995) Steroid elution improves the stimulation threshold in an activefixation atrial permanent pacing lead. Circulation, 92(10), 2935-2939.

[5] Rao, C.R. (1997) Linear statistical inference and its applications. Wiley \& Sons, New York. 